\title{
Fast adiabatic method for measuring topological Chern number
}

\author{
ZhiHuang Luo ${ }^{1,2}$, and Bei Zeng ${ }^{2,3 *}$ \\ ${ }^{1}$ Beijing Computational Science Research Center, Beijing 100193, China; \\ ${ }^{2}$ Institute for Quantum Computing and Department of Physics and Astronomy, University of Waterloo, Waterloo N2L 3G1, Canada; \\ ${ }^{3}$ Department of Mathematics and Statistics, University of Guelph, Guelph N1G 2W1, Canada \\ Received January 24, 2018; accepted January 26, 2018; published online February 26, 2018
}

Citation: Z. H. Luo, and B. Zeng, Fast adiabatic method for measuring topological Chern number, Sci. China-Phys. Mech. Astron. 61, 057431 (2018), https://doi.org/10.1007/s11433-018-9173-5

Topological phases play an increasingly central role in condensed matter physics $[1,2]$ and fault-tolerant quantum computation [3]. The global nature can be characterized by certain topological invariants, many among them can be defined as the integrals of some geometric quantities. A well-known example is the Chern number [4]. It is the integral of Berry curvature over a surface without boundary and is thus closely related to Berry phase [5]. The integer Chern number can be interpreted as the filling factor emerging in quantized plateaus of the Hall conductance of 2D electronic systems [6]. As a whole property of parameter space, the Chern number reveals the degenerate points of ground states, which can be viewed as magnetic monopoles from the electromagnetic analogy. We can use the Chern number to probe different topological phases and their corresponding transitions.

However, the measurement of Chern number is a extremely challenging, due to its globally topological property. The previous works usually relied on the adiabatic interference [7,8], but this method is limited to systems of weakly interacting quasi-particles. Since the adiabatic evolution is time-consuming especially for a complicated interacting quantum system, it is hard to achieve before quantum coherence is destroyed. An alternative method to direct measurement was proposed by Gritsev et al. [9], which states that Berry curvature can be extracted via the linear response of local physical observables to the rate of change of an external

\footnotetext{
*Corresponding author (email: zengb@uoguelph.ca)
}

parameter. Along the lines of this idea, some experiments were performed to observe topological transitions using oneor two- superconducting qubits $[10,11]$. More recently, the experiment has been extended to four-nuclear-spin systems [12]. The direct measurement of Berry curvature takes advantage of linear term in the response, and only need to change the parameters slowly, but not necessarily adiabatically. It provides a general means to probe topological properties.

In a recent experiment, Wang and his collaborators [13] applied the "shortcut to adiabaticity" (STA) protocol $[14,15]$ to measure the Chern number, and simulated the topological transition of the Su-Schrieffer-Heeger model using a superconducting qubit. In the STA protocol, an additional counterdiabatic Hamiltonian was designed to satisfy that there is no non-adiabatic transitions of the ground state, which speed up the adiabatic evolution of original Hamiltonian and achieve the fast adiabatic state transfer. The schematic diagram of fast adiabatic trajectory for measuring Chern number is illustrated in Figure 1. To reduce the influence of higher excited states, Wang and his collaborators incorporated the technique of deviation removal by adiabatic gates into the fast adiabatic trajectory. They performed the experimental simulation of topological transitions in both real-time and virtual ways. The virtual way of constructing the fast adiabatic trajectory provides the feasibility of mimicking the first Brillouin zone. Their work successfully demonstrated the application of fast adiabatic method towards topological systems. 


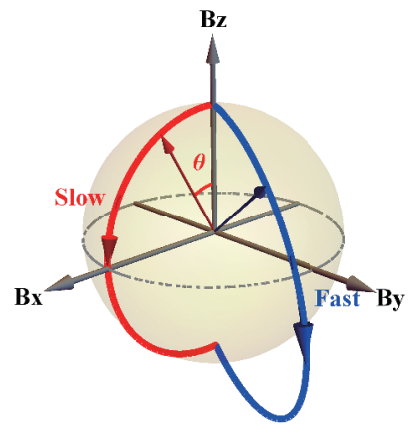

Figure 1 (Color online) Schematic diagram of fast adiabatic trajectory (blue) in the parameter space of a drive magnetic field for measuring topological Chern number. It includes an additional counter-diabatic field, which do not lead to non-adiabatic transitions of a quantum state but speed up the measurement. The red curve represents the slow adiabatic evolution of original Hamiltonian without the counter-diabatic field.

1 D. C. Tsui, H. L. Stormer, and A. C. Gossard, Phys. Rev. Lett. 48 , 1559 (1982).

2 X. G. Wen, and Q. Niu, Phys. Rev. B 41, 9377 (1990).

3 A. Y. Kitaev, Ann. Phys. 303, 2 (2003).
4 S. Chern, Ann. Math. 47, 85 (1946).

5 M. V. Berry, Proc. R. Soc. A-Math. Phys. Eng. Sci. 392, 45 (1984).

6 K. Klitzing, G. Dorda, and M. Pepper, Phys. Rev. Lett. 45, 494 (1980).

7 X. Peng, S. Wu, J. Li, D. Suter, and J. Du, Phys. Rev. Lett. 105, 240405 (2010).

8 P. J. Leek, J. M. Fink, A. Blais, R. Bianchetti, M. Goppl, J. M. Gambetta, D. I. Schuster, L. Frunzio, R. J. Schoelkopf, and A. Wallraff, Science 318, 1889 (2007).

9 V. Gritsev, and A. Polkovnikov, Proc. Natl. Acad. Sci. 109, 6457 (2012).

10 M. D. Schroer, M. H. Kolodrubetz, W. F. Kindel, M. Sandberg, J. Gao, M. R. Vissers, D. P. Pappas, A. Polkovnikov, and K. W. Lehnert, Phys. Rev. Lett. 113, 050402 (2014).

11 P. Roushan, C. Neill, Y. Chen, M. Kolodrubetz, C. Quintana, N. Leung, M. Fang, R. Barends, B. Campbell, Z. Chen, B. Chiaro, A. Dunsworth, E. Jeffrey, J. Kelly, A. Megrant, J. Mutus, P. J. J. OMalley, D. Sank, A. Vainsencher, J. Wenner, T. White, A. Polkovnikov, A. N. Cleland, and J. M. Martinis, Nature 515, 241 (2014).

12 Z. Luo, C. Lei, J. Li, X. Nie, Z. Li, X. Peng, and J. Du, Phys. Rev. A 93, 052116 (2016).

13 T. Wang, Z. Zhang, L. Xiang, Z. Gong, J. Wu, and Y. Yin, Sci. ChinaPhys. Mech. Astron. 61, 047411 (2018).

14 M. Demirplak, and S. A. Rice, J. Phys. Chem. A 107, 9937 (2003).

15 X. Chen, I. Lizuain, A. Ruschhaupt, D. Guéry-Odelin, and J. G. Muga, Phys. Rev. Lett. 105, 123003 (2010). 